Thorax, 1980, 35, 355-358

\title{
Comparison of serial electrocardiographic and vectorcardiographic changes during recovery from status asthmaticus
}

\author{
J R M BATEMAN, D SIEGLER, D WAGSTAFF, AND S W CLARKE \\ From the Departments of Thoracic Medicine and Cardiology, The Royal Free Hospital, London
}

\begin{abstract}
Serial electrocardiographs (ECG) and vectorcardiographs (VCG) have been performed on 10 patients admitted to hospital in status asthmaticus on 12 separate occasions. The VCG was more efficient than the ECG in the detection of right atrial and ventricular enlargement. Both investigations were equally reliable in recording changes in frontal plane $P$ wave axis. The mean frontal plane $\mathrm{P}$ wave axis fell from $+60^{\circ}$ (range $+35^{\circ}$ to $+90^{\circ}$ ) on the day of admission to $+43^{\circ}$ (range $+30^{\circ}$ to $+60^{\circ}$ ) at the time of discharge. The mean $\mathrm{FEV}_{1}$ expressed as a percentage of predicted values increased from $48 \%$ (range $25 \%$ to $81 \%$ ) to $87 \%$ (range $44 \%$ to $123 \%$ ). In direct contrast to previous studies the presence of an abnormally vertical frontal plane $\mathrm{P}$ wave axis $\left(>60^{\circ}\right)$ was related to the severity of airway obstruction $(\mathrm{p}<0.01)$.
\end{abstract}

Reversible changes in the electrocardiograph (ECG) during a severe acute asthma attack have previously been described. ${ }^{12}$ These changes, however, have not appeared to correlate with the severity of airways obstruction and furthermore, have been based upon retrospective studies. The present study was designed to follow cardiac changes in a precise and serial manner during recovery from severe acute asthma, using the non-invasive techniques of electro-and vectorcardiography and the attempt to relate these changes to the severity of airway obstruction.

\section{Methods}

Ten patients, five men and five women with a mean age of 30 years (range 16-58 yr) have been studied during 12 separate admissions to hospital for treatment of severe acute asthma persisting for more than 24 hours. Simple pulmonary function tests (forced expiratory volume in one second $\left(\mathrm{FEV}_{1}\right)$, forced vital capacity (FVC), and peak expiratory flow rate (PEFR)), arterial blood gases, plasma urea and electrolytes, a standard 12 lead ECG, and a vectorcardiograph using the Frank system ${ }^{3}$ with the patient semi-supine, were performed at the time of admission, at the time of discharge, and at an intermediate time during

Address for reprint requests: Dr JRM Bateman, East Birmingham Hospital, Bordesley Green East, Birmingham B9 5ST. the hospital stay. All patients received intensive therapy consisting of intravenous corticosteroids and bronchodilators, aerosol bronchodilators, oxygen, chest physiotherapy, and antibiotics. Both the ECG and VCG were examined particularly for the presence of right atrial and ventricular enlargement.

For the ECG we adopted the criteria as used by Siegler. " "Right atrial enlargement" was present if the height of the $P$ wave in standard leads II and III exceeded $2.5 \mathrm{~mm}$ and "right ventricular enlargement" if there was right axis deviation to beyond $+110^{\circ}$, the height of the $R$ wave in $V_{1}$ exceeded $5 \mathrm{~mm}$, and the ratio of the $R$ wave amplitude to $S$ wave amplitude in $V_{1}$ exceeded unity. ${ }^{4}$ The criteria described by Chou et $a l^{5}$ were used in the analysis of the VCG. In right atrial enlargement the spatial $P$ loop was directed inferiorly and to the left with most of the loop located anteriorly. For right ventricular enlargement one or more of the following criteria were satisfied: (1) in the frontal plane the QRS loop area in the right inferior quadrant was less than $30 \%$ of the total; (2) in the transverse plane the QRS loop area in the left posterior quadrant was less than $30 \%$ of the total; (3) in the transverse plane the QRS loop area in the right posterior quadrant was greater than $20 \%$ of the total.

The frontal plane $P$ wave axis was measured 
using the hexagonal reference system on the ECG and directly on the VCG. An axis greater than $+60^{\circ}$ was taken to be abnormal. ${ }^{\circ}$ As the number of patients studied is small and the results do not follow a normal distribution, Wilcoxon's methods for paired and unpaired comparisons have been used for the statistical analysis. ${ }^{7}$

\section{Results}

On admission vectorcardiography demonstrated the presence of right atrial enlargement in seven cases. Right ventricular enlargement of the $C$ type $^{5}$ was found in four cases. As examples, fig 1 shows normal QRS vectorcardiograph loops from

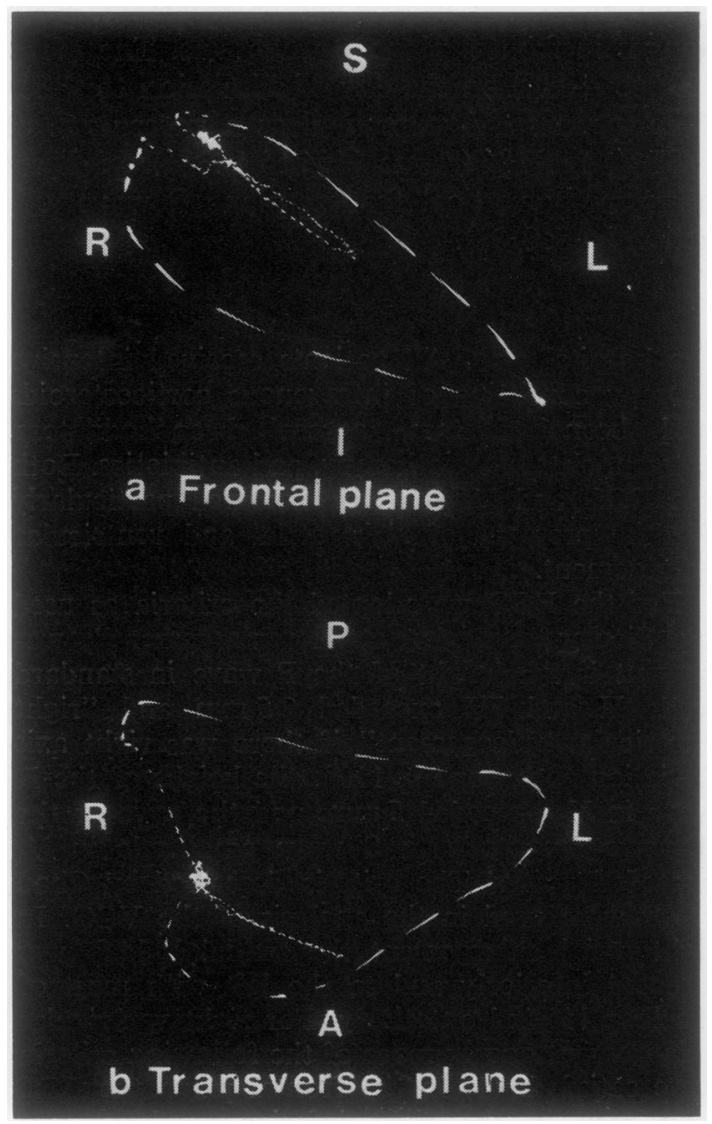

Fig 1 Normal vectorcardiograph in two conventional planes from a patient who had recovered from severe acute asthma. The two planes are (a) frontal and (b) transverse. $S=$ superior, $I=$ inferior, $R=$ right,$L=$ left,$P=$ posterior, $A=$ anterior. Note the smaller $P$ and $T$ loops within the larger $Q R S$ loop. The direction of the inscription of each loop is shown by the rounder end of each elongated dash. Each dash represents $2.5 \mathrm{~ms}$. a patient who had recovered from severe acute $\overrightarrow{\vec{s}}$ asthma, and fig 2 abnormal loops from a patient $-\overrightarrow{0}$ at the time of admission with severe acute asthma. Electrocardiography was unable to $\overline{\frac{\bar{\sigma}}{5}}$ demonstrate right ventricular enlargement and $\vec{\nabla}$ demonstrated right atrial enlargement in only three cases. At the time of discharge all the ECGs were normal, but one VCG demonstrated $\vec{O}$ persistence of right atrial enlargement. Because $\overrightarrow{\vec{\omega}}$ of technical difficulties and the severity of asthma at the time of admission we were unable to $\vec{z}$ examine the effect of respiratory phase on the $\underset{\omega}{x}$ VCG tracings. Careful scrutiny of the ECG on

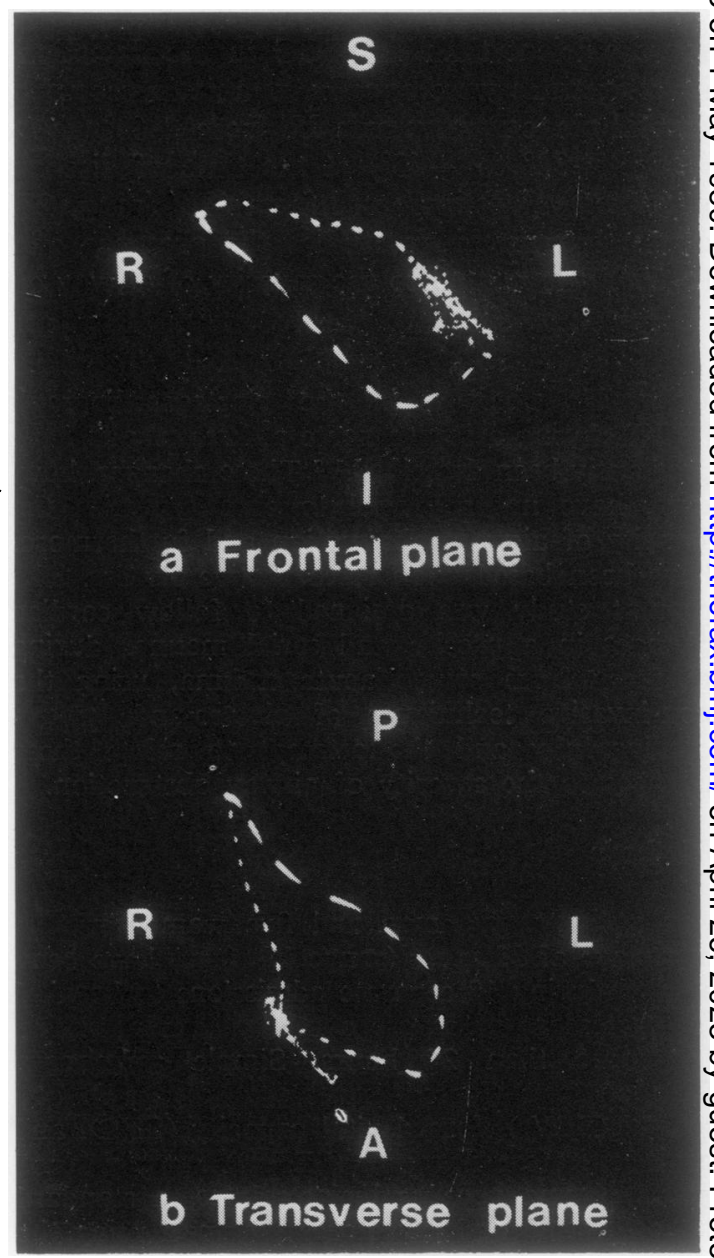

Fig 2 Abnormal vectorcardiograph from a patient at the time of admission to hospital with severe acute asthma. In the frontal plane the QRS loop lies mainly inferiorly and to"the right, and in the transverse plane largely posteriorly. 
tracings, however, failed to demonstrate significant differences in our measurements resulting from this variable.

There was no disparity between the VCG and ECG in the measurement of frontal plane $P$ wave axis. The mean FEV, FVC, PEFR, and the frontal plane $\mathrm{P}$ wave axis for 10 patients at admission and discharge are shown in the table 1. There was a significant improvement in all pulmonary function indices accompanied by a significant fall in frontal plane $P$ wave axis $(\mathrm{p}<0 \cdot 01)$.

An abnormal frontal plane $P$ wave axis $\left(>+60^{\circ}\right)$ was found in both the VCG and ECG in five patients at the time of admission. This reverted to normal by the time of discharge. The mean FEV, FVC, and PEFR in the presence of firstly an abnormal frontal plane $P$ wave axis and secondly a normal axis is shown in table 2 . All the parameters of pulmonary function were significantly less when this axis was abnormal $(\mathrm{p}<0.01)$.

Abnormalities in neither the VCG nor the ECG could be correlated with arterial blood gas analysis. Furthermore, plasma electrolyte values were invariably normal on admission and throughout the hospital stay.

\section{Discussion}

Our results clearly demonstrate the following

Table 1 Simple pulmonary function indices (expressed as a percentage of predicted values) and frontal plane $P$ wave axis (degrees) for 10 patients at the time of admission to and discharge from hospital

\begin{tabular}{llll}
\hline $\begin{array}{l}\text { P.lmonary } \\
\text { unction indices }\end{array}$ & & On admission & On discharge \\
\hline FEV $_{1}$ & Mean & 48 & 87 \\
& Range & $25-81$ & $44-123$ \\
FVC & Mean & 67 & 98 \\
& Range & $36-111$ & $65-127$ \\
PEFR & Mean & 43 & 78 \\
& Range & $22-77$ & $52-123$ \\
Frontal plane & Mean & +60 & +43 \\
P wave axis & Range & $+35-+49$ & $+30-+60$ \\
\hline
\end{tabular}

Table $2 F E V_{1}, F V C$, and PEFR (expressed as a percentage of predicted values) in the presence of a frontal plane $P$ wave axis $>+60^{\circ}$ and $<+60^{\circ}$

\begin{tabular}{llll}
\hline & & \multicolumn{2}{c}{ Frontal plane $P$ wave axis } \\
\cline { 3 - 4 } & & $>+60^{\circ}$ & $<+60^{\circ}$ \\
\hline FEV $_{1}$ & Mean & 42 & 77 \\
& Range & $24-53$ & $25-125$ \\
FVC & Mean & 61 & 94 \\
& Range & $36-86$ & $46-122$ \\
PEF R & Mean & 33 & 72 \\
& Range & $22-40$ & $24-133$ \\
\hline
\end{tabular}

points. Firstly, the vectorcardiograph is more sensitive than the electrocardiograph in the detection of right atrial and ventricular enlargement. Secondly, both methods are equally efficient for the measurement of frontal plane $P$ wave axis. Thirdly, the frontal plane $P$ wave axis decreases with relief of the airways obstruction, and finally an abnormal axis is related to the severity of airway obstruction. With the exception of the persistence of right atrial enlargement on the VCG, but not on the ECG, in one patient all the observed cardiographic abnormalities disappeared after bronchodilatation and resolution of the attack.

Abnormalities previously noted on the ECG have included right axis deviation, prominent $P$ waves, inversion of $T$ waves, right bundle branch block, and ST segment changes. On the VCG abnormalities noted have included right atrial and ventricular preponderance, rotation of the QRS loop, posterior "pip" pattern, and first degree $\mathrm{T}$ loop changes in the presence of airways obstruction. ${ }^{12}$ 8-18 $^{-1}$ It is well known that the VCG is more sensitive than the ECG in the detection of cardiographic abnormalities, ${ }^{9} 121317$ and resolution of abnormalities after the reversal of acute airway obstruction has been reported using either method, but not both simultaneously. ${ }^{121011}$ Rao et $a l^{13}$ compared both methods in children, firstly during acute asthma and secondly when in remission, but not during the recovery period. The present study, however, is the first to compare directly the efficiency of both methods in the detection and resolution of cardiographic abnormalities in a serial manner during recovery from severe acute asthma.

With one exception previous workers have been unable to relate cardiographic abnormalities to the severity of airway obstruction. Chapman ${ }^{8}$ studied 1144 patients with chronic non-specific lung disease and demonstrated a good correlation between a vertical $P$ wave axis in the ECG and the degree of airway obstruction. Siegler ${ }^{2}$ found a high incidence $(78 \%)$ of an abnormally vertical $P$ wave axis in the ECG of patients with acute asthma, but was unable to correlate this with the severity of airway obstruction. We have thus examined closely the relationship between the frontal plane $P$ wave axis in both the ECG and VCG, and simple measurements of lung function. We have been able to demonstrate firstly a significant reduction in the frontal plane $P$ wave axis accompanied by a significant improvement in lung function and secondly that an abnormal axis is related to the degree of airway obstruction.

Although the mechanism of $P$ wave changes 
in lung disease is not clear, there seems to be fairly general agreement that the phenomenon is associated with overinflation of the lungs. ${ }^{8}$ Our findings confirm this view. Furthermore, the lack of correlation with arterial blood gas analysis and serum electrolyte values gives additional support to Siegler's postulation ${ }^{2}$ that such cardiographic changes are explicable on purely anatomical grounds-because of overdistension of the lungs the position of the heart is altered resulting in changes in electrical axis.

\section{References}

1 Rebuck AS, Read J. Assessment and management of severe asthma. Am J Med 1971; 51:78898.

2 Siegler D. Reversible electrocardiographic changes in severe acute asthma. Thorax 1977; 32:328-32.

3 Frank E. An accurate, clinically practical system for spatial vectorcardiography. Circulation 1956; 13:737-49.

4 Estes EH Jr. The ventricular electrocardiogram. In: Hurst JW, Logue RB, Schlant RC, Wenger NK (eds). The Heart. Third edition. New York: McGraw-Hill, 1974:308.

5 Chou TC, Helm RA, Kaplin S. Clinical vectorcardiography. Second edition. New York: Grune and Stratton, 1974.

6 Schamroth L. An introduction to electrocardiography. Fifth edition. Oxford: Blackwell, 1977.

7 Snedecor GW, Cochran WG. Statistical methods. Sixth edition. Ames, Iowa: Iowa State University Press, 1968.

8 Chapman TT. Lung function tests and a "vertical" $P$ wave axis in the electrocardiogram. $\stackrel{\overrightarrow{\bar{D}}}{\stackrel{\vec{\rho}}{\vec{\rho}}}$ Thorax 1974; 29:106-9.

9 Criep LH, Silverblatt M. The effect of bronchial 흔 asthma on the heart with special reference to $\frac{\bar{D}}{}$ the spatial vectorcardiogram. J Allergy 1960; $\mathbb{\mathbb { Q }}$ 31:191-210.

10 Da Costa JL, Chia BL. The electrocardiographic की changes in bronchial asthma and their relation- $\vec{O}$ ship to the severity of airways obstruction. Singapore Med J 1974; 15:101-5.

11 Gunstone RF. Right heart pressures in bronchial asthma. Thorax 1971; 26:39-45.

12 Quivers WW, Linde LM, Sapin SO, Heimlich w EM. The electrocardiogram and vectorcardio-ir gram in children with severe bronchial asthma. Am J Cardiol 1964; 14:616-21.

13 Rao M, Steiner P, Paydar M, Padre R, Torres 9 RR. Electrophysiological changes in the heart of children with bronchial asthma during the acute attack and later during remission. J Asthma Res 1974; 11:113-21.

14 Shmock CL, Mitchell RS, Pomerantz B, Pryor $\vec{\oplus}$ R, Maisel JC. The electrocardiogram in chronic. airway obstruction. Chest 1971; 60:335-40.

15 Spodick DH. Electrocardiographic studies in pulmonary disease. Circulation 1959; 20:1067-74.

16 Spodick DH. Vectorcardiogram in pulmonary emphysema: its relationship to scalar electro- $\mathbb{D}$ cardiographic findings. Am Rev Respir Dis 1968; 98:634-9.

17 Stern EA, Tenney SM. Correlation of the spatial vectorcardiogram and electrocardiogram in right ventricular hypertrophy. Am Heart $J$ 1956; 51:53-60.

18 Walsh TJ. The vectorcardiogram in cor pulmonale. Prog Cardiovasc Dis 1967; 9:363-81. 